\title{
Intact verbal description of letters with diminished awareness of their forms
}

\author{
Kyoko Suzuki, Atsushi Yamadori
}

\begin{abstract}
Visual processing and its conscious awareness can be dissociated. To examine the extent of dissociation between ability to read characters or words and to be consciously aware of their forms, reading ability and conscious awareness for characters were examined using a tachistoscope in an alexic patient. A right handed woman with 14 years of education presented with incomplete right hemianopia, alexia with kanji (ideogram) agraphia, anomia, and amnesia. Brain MRI disclosed cerebral infarction limited to the left lower bank of the calcarine fissure, lingual and parahippocampal gyri, and an old infarction in the right medial frontal lobe. Tachistoscopic examination disclosed that she could read characters aloud in the right lower hemifield when she was not clearly aware of their forms and only noted their presence vaguely. Although her performance in reading kanji was better in the left than the right field, she could read kana (phonogram) characters and Arabic numerals equally well in both fields. By contrast, she claimed that she saw only a flash of light in $61 \%$ of trials and noticed vague forms of stimuli in $36 \%$ of trials. She never recognised a form of a letter in the right lower field precisely. She performed judgment tasks better in the left than right lower hemifield where she had to judge whether two kana characters were the same or different. Although dissociation between performance of visual recognition tasks and conscious awareness of the visual experience was found in patients with blindsight or residual vision, reading (verbal identification) of characters without clear awareness of their forms has not been reported in clinical cases. Diminished awareness of forms in our patient may reflect incomplete input to the extrastriate cortex.
\end{abstract}

(F Neurol Neurosurg Psychiatry 2000;68:782-786)

Keywords: blindsight; alexia; consciousness; letter discrimination

Dissociation between a cognitive processing and conscious awareness of the processing is one of the stimulating topics of neurology, but neural mechanisms underlying the conscious awareness remained unsolved. ${ }^{12}$ This dissociation was most often reported in visual processing. For example, lesions affecting the primary visual cortex (V1) can uncouple visual discrimination and awareness. A patient with the V1 lesion sometimes showed discrimination of visual stimuli without conscious awarenessthat is, blindsight. ${ }^{3-6}$ In addition, recent studies reported residual vision that was not always associated with clear awareness of stimuli in patients with striatal lesions..$^{7-12}$ In these studies, awareness for visual stimuli was examined by methods which require minimal verbal expression, such as forced choice responses about a property or yes/no detection paradigm. On the other hand, verbal identification of stimuli without clear awareness of their forms has not been reported in clinical cases. We report on a patient with alexia who could read characters aloud presented to the right lower quadrant field without clear consciousness of their forms and we attempt to elucidate neural mechanisms of conscious awareness.

\section{Case report}

A 48 year old right handed woman with 14 years of education was admitted to a hospital because of an inability to read. The patient had experienced transient weakness of her right leg 3 years before and at the same time an aneurysm of the anterior communicating artery was incidentally found and clipped. One and half months later, the patient was referred to our hospital for rehabilitation. Neurological examination on admission showed incomplete right homonymous hemianopia, slight right hemiparesis, alexia, anomia, and amnesia. Other cranial nerves and cerebellar function were intact. Deep tendon reflexes were symmetric without pathological reflexes. There was no visual agnosia or prosopagnosia. A Humphrey visual analyser demonstrated right homonymous hemianopia sparing a part of the lower medial field (fig 1). Corrected visual acuity was normal. Brain MRI showed a cerebral infarction in the left lingual and parahippocampal gyri and a part of the splenium, and an old lesion in the right medial frontal area associated with the previous clipping procedure (fig 2). Blood flow measured with ${ }^{123} \mathrm{I}-$ IMP single photon emission tomography 
(SPECT) showed a decrease in blood flow in the territory of the left posterior cerebral artery and in the right medial frontal lobe. A whole head magnetometer combined with MRI showed a visual evoked field (P100m response) in the right medial occipital lobe corresponding to the stimuli in the left visual field, but no clear responses to those in the right visual field. The following neuropsychological evaluation was performed during the next 2 months. All
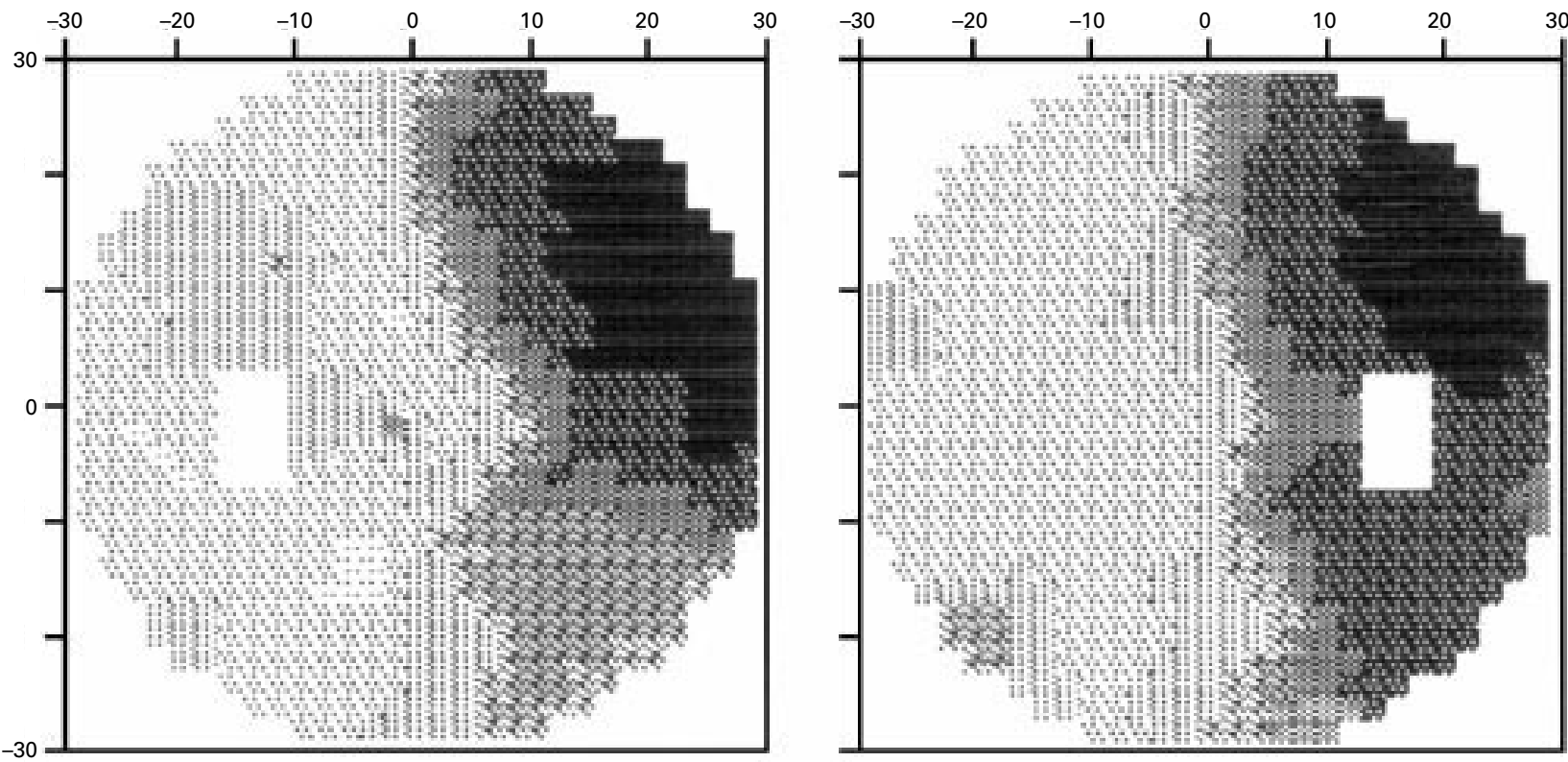

Figure 1 Visual fields assessed with a Humphrey visual analyser showing right homonymous hemianopia with spared region in the right medial lower field.

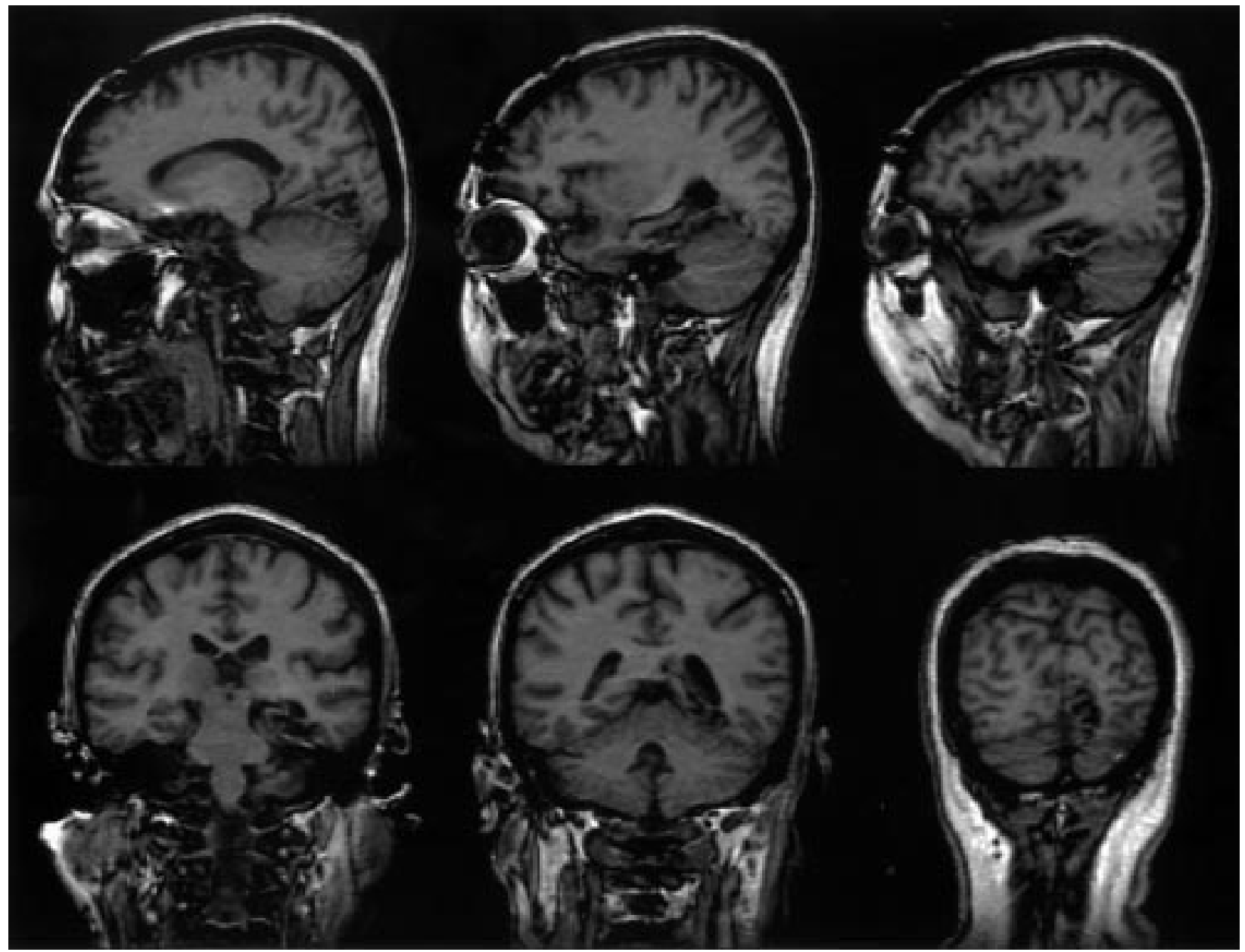

Figure 2 T1 weighted MR images demonstrated cerebral infarction in the left striate, lingual, and parahippocampal gyri and a part of the splenium. 
experimental investigations were performed in compliance with the relevant laws and institutional guidelines. She gave informed consent before the examination.

READING ABILITY AND AWARENESS RESPONSES Systematic neuropsychological examination was performed to show the nature of her deficits. She was alert, cooperative, and fully oriented all the time. Language was evaluated by the standard language test for aphasia in Japanese (SLTA), which was developed to examine various aspects of language ability for native Japanese speakers and has been used as a standard test for more than 20 years in Japan. Her overall performance on the SLTA was normal except for anomia and severe alexia with kanji (ideogram) agraphia. Her verbal utterances were fluent without any paraphasia. Her comprehension of spoken speech and repetition was good. She made seven errors out of 20 in confrontation naming. Writing was preserved for two types of hiragana (phonogram) and simple kanji characters but not for complex kanji characters. We defined simple kanji characters as those learnt by 9 years of age at a primary school, most of which consist of less than seven or eight strokes. She was significantly impaired in the reading aloud and reading comprehension subtests. In the reading aloud task, she made three errors out of 10 for kana and kanji words and two errors for Arabic numerals. She used letter by letter strategy and her time for reading was prolonged as the number of characters increased. In the Wechsler adult intelligence scale-revised, her verbal IQ was 94, and her performance IQ was 62. A substantial memory deficit was evidenced on the Wechsler memory scale-revised. The patient had a verbal memory index of 58, a visual memory index of 92 , and a general memory index of 61. In summary, her neuropsychological impairment included alexia characterised by letter by letter reading, mild agraphia for kanji, anomia, and amnesia for verbal material. Her ability to read hiragana and kanji words had not changed markedly

Tachistoscopic examination and awareness responses in each visual field

\begin{tabular}{|c|c|c|}
\hline & L lower quadrant field & $R$ lower quadrant field \\
\hline \multicolumn{3}{|l|}{ Task 1: Pointing } \\
\hline Kana letters & $25 / 32$ & $23 / 32$ \\
\hline Kanji letters & $28 / 32$ & $25 / 32$ \\
\hline Arabic numerals & $27 / 32$ & $26 / 32$ \\
\hline Kana words & $4 / 8$ & $5 / 8$ \\
\hline Objects & $8 / 8$ & $4 / 8$ \\
\hline Colours & $2 / 8$ & $4 / 8$ \\
\hline Patternst & $7 / 8$ & $5 / 8$ \\
\hline Task 2: Judgement of Kana letters $\star$ & $22 / 24$ & $15 / 24$ \\
\hline \multicolumn{3}{|l|}{ 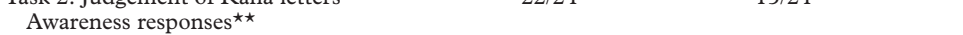 } \\
\hline Unaware: no feeling of something being there & $0 / 24(0.0 \%)$ & $0 / 24(0.0 \%) \ddagger$ \\
\hline Vague: a feeling of a flash of light & $0 / 24(0.0 \%)$ & $17 / 24(58.8 \%)$ \\
\hline Vague recognition of the form & $2 / 24(50.0 \%)$ & $7 / 24(71.4 \%)$ \\
\hline Aware: certain of the form & $22 / 24(95.5 \%)$ & $0 / 24(0.0 \%)$ \\
\hline \multicolumn{3}{|l|}{ Task 3: Read aloud } \\
\hline Kana letters & $23 / 24$ & $21 / 24$ \\
\hline Kanji letters $\star \star$ & $22 / 24$ & $13 / 24$ \\
\hline Arabic numerals & $11 / 24$ & $17 / 24$ \\
\hline Total correct responses & $56 / 72$ & $51 / 72$ \\
\hline \multicolumn{3}{|l|}{ Awareness responses $\star \star$} \\
\hline Unaware: no feeling of something being there & $0 / 72$ & $2 / 72(0.0 \%) \ddagger$ \\
\hline Vague: a feeling of a flash of light & $0 / 72$ & $44 / 72(70.5 \%)$ \\
\hline Vague recognition of the form & $5 / 72(20.0 \%)$ & $26 / 72(76.9 \%)$ \\
\hline Aware: certain of the form & $67 / 72(82.1 \%)$ & $0 / 72$ \\
\hline
\end{tabular}

${ }^{\star} \mathrm{p}<0.05 ;{ }^{\star \star} \mathrm{p}<0.005$. $†$ Meaningless patterns with several straight and curved lines. during her stay, as confirmed by comparable results of a shortened version of reading tests performed in the 6 th and 10 th week.

We further investigated her reading ability in both visual hemifields to clarify her visual recognition and conscious awareness for it in the 9th week postonset (table). Tachistoscopic examination required the patient to sit $50 \mathrm{~cm}$ away from a high resolution red-green-blue colour monitor of a Power MacIntosh 6200/75 computer, with her eyes aligned with a fixation cross in the middle of the screen. An examiner always monitored her eyes to ensure that she was fixating the cross during stimulus presentation. A stimulus, the width of which was a visual angle of 1 to 2 degrees, appeared for 100 $\mathrm{ms}$ in the right or left lower visual field at a visual angle of 2 to 3 degrees from the vertical meridian. Thus stimuli were presented in the area where visual stimuli with increased luminance, about $10 \mathrm{~dB}$ above those in the intact left visual field, could be detected. Her ability to match, discriminate, and read stimuli was examined. Seven types of stimuli were used for matching; one hiragana letter, one kanji letter that corresponded to a concrete word, one or a pair of Arabic numerals, a hiragana word that consisted of two characters and corresponded to the same concrete word as the kanji letter stimulus, a line drawing of an object, colour, and a meaningless pattern with several straight and curved lines. The complexity of meaningless patterns was almost equal to that of kanji characters. She was asked to point to the same one as a stimulus out of eight items from the same category just after the stimulus presentation (task 1). Discriminative function was also measured in which the patient was asked whether two kana characters vertically presented in one lower field were same or different (task 2). Her ability to read characters was examined using three types of stimuli: kanji characters, kana characters, and Arabic numerals. She was requested to read a letter aloud just after the stimulus presentation (task 3). During tasks 2 and 3, she was asked what she saw and how clear the stimulus was and she answered orally. There were 48 trials for each type of stimulus, randomly presented in both visual fields (half of the trials in each field) in tasks 2 and 3. Task 3 was repeated on two separate days to confirm the reproducibility of the data.

In task 1, her performance on matching for each stimulus group was not significantly different between two lower quadrant fields $\left(\chi^{2}\right.$, $\mathrm{p}>0.05)$. In task 2 , judging ability in both visual fields was different from each other $\left(\chi^{2}\right.$, $\mathrm{p}<0.05)$. Her awareness responses for stimuli in the right lower visual field were, however, more significantly impaired compared with those in the left lower field (Mann-Whitney test, $\mathrm{p}<0.005)$. In 17 out of 24 trials, she denied having seen any form but said that she saw a flash of light in the right field. In task 3, although reading kanji characters was significantly impaired in the right lower visual field $\left(\chi^{2}, \mathrm{p}<0.005\right)$, she could read kana characters and Arabic numerals at the same level in both visual fields $\left(\chi^{2}, p>0.05\right.$; table). By contrast, she declared that she saw only a flash of light in 44 
out of 72 trials although she read them aloud correctly in $70 \%$ of trials. Her awareness responses for stimuli in the right lower visual field were significantly lower than those in the left lower field (Mann-Whitney test, $\mathrm{p}<0.005)$. She often declared that she could not understand how it was possible for her to read the letter aloud when she saw only a flash of light. The ratio of correct responses in the right lower field was not different between the trials when she saw only a flash of light and those when she was aware of vague forms of characters.

\section{Discussion}

The present data demonstrate a clear dissociation between our patient's ability to read characters and her ability to be aware of their forms. She was able to extract "verbal" information of a stimulus without clear awareness of its intrinsic visual information - that is, form. We think that this is the first clinical report that processing of complex visual pattern-like characters can occur without clear visual awareness of their form. Dissociation between visual awareness and visual recognition was reported in normal volunteers by modulating stimuli. Adams showed that with very brief, masked presentation subjects could identify orthographic stimuli without being able to report accurately the case or font of their presentation. ${ }^{13}$ Normal subjects could report the location of a stimulus at better than chance accuracy when they claimed no form perception, because of short exposure duration and pattern masking. ${ }^{14}$ By superimposing complementary textures, such as opposite motion or binocular rivalry and orthogonal orientation, normal subjects could locate the texture contrast reliably but not consciously experience it. ${ }^{15}$

Previous studies suggested uncoupling between visual awareness and discrimination in cases with cortical blindness or hemianopia,that is, blindsight. Patterns of impaired and preserved abilities in cases of blindsight show considerable variability from case to case. Most patients could detect and localise a light. ${ }^{10}$ Some patients could discriminate orientation and shape, and detect movement and flicker. ${ }^{31617}$ Patients with blindsight have usually no phenomenal experience of seeing a processed stimuli at all; but some may experience a type of contentless awareness or a feeling of something happening. Total unawareness and a vague feeling of a stimulus may be experienced, as reported in patient GY who demonstrated a marked variability in his awareness for a given level of discrimination of motion direction. ${ }^{18}$ In addition, residual vision in patients with striatal lesions was not associated with relevant awareness for visual stimuli. Blythe et al reported patients with residual vision who demonstrated ability to localise visual target by eye movements and hand reaching when they experienced a transient shadow in the "blind" field and could not identify any characteristic of the stimulus. ${ }^{10}$ Patient DB could report horizontal or vertical lines and discriminate $\mathrm{X}$ or $\mathrm{O}$ above chance level. During these experiments his most com- mon response was that he saw nothing at all, but, if pressed, he said that he had a "feeling" that the stimulus was either pointing this or that way and smooth (the O) or jagged (the $\mathrm{X}) .^{7}$ Marcel reported on two patients with homonymous right hemianopia who demonstrated conscious and non-conscious perception of shape in the blind field. ${ }^{19}$ Their conscious visual experience was, however, only a dark shadow or a flash, not shape. In summary, patients with blindsight or residual vision sometimes felt or noticed the presence of stimuli, but this vague awareness was uncoupled with the visual processing that was actually done during tasks.

Dissociation between visual processing and conscious awareness in our patient was similar to these patients in a sense that conscious awareness was not enough for the task to be solved. But our patient was different in that she showed descriptive ability of characters when she was aware of stimulus presentation, but not clearly aware of the form. In other words, she was somehow able to extract the essential attribute of a stimulus - that is, its name-but was unable to consciously recognise its form and had no idea whether the answer was correct or not. Her responses were not mere uncertainty about the answer, but she could not tell whether they had any certain form and saw a flash of light instead. In reported patients with residual vision whose lesions were confirmed in the striate and extrastriate cortices, they could discriminate one of two shapes or characters but could not describe characters verbally. Patient GY reported some sort of form when presented with words like "waves" which were straight or curved, or a "squareness", but could not read the words. ${ }^{6}$ Patients DB and TP answered "smooth" or "jagged" in letter discrimination tasks when pressed to say, but they could not name the letter. ${ }^{719}$

Lesions in our patient involved the striate and peristriate cortex below the calcarine fissure in the left hemisphere. The left lingual gyrus was included in the lesions, but the fusiform gyrus was spared. A patient with lesions in the right lower bank of the calcarine fissure and the lingual gyrus demonstrated unconscious visual processing in the left upper field that was related to the remaining islands of vision from preserved striate cortex. ${ }^{12}$ It is assumed that people may not be aware of some of the neuronal activity in the V1. ${ }^{1}$ Patients who hallucinate have increased ventral extrastriate activity which persists between hallucinations. ${ }^{20}$ They found that the rise in functional magnetic resonance imaging (fMRI) occurred before the onset of the conscious visual experience (hallucination) without visual input. The possible explanation may be that cerebral activity has to exceed a certain threshold level to contribute to visual consciousness. A study using fMRI for patient GY demonstrated that there was a relation between conscious awareness of motion and cerebral activity in area V5 and in the reticular activating system. ${ }^{18}$ Thus activity in the extrastriate cortex above some threshold might be necessary to acquire conscious visual processing. Recent 
neuroimaging and neurophysiological studies showed the importance of the fusiform gyrus for reading. ${ }^{21}{ }^{22}$ In our patient we speculate that the spared left fusiform gyrus received enough visual information about characters to read them aloud, probably via the residual V1 (cuneus), but the amount of activity in the extrastriate cortex might not have been sufficient to evoke conscious experience of their forms.

This research was partly supported by grants (No 10557059 and No 09770436 to KS) from the Ministry of Education, Science and Culture, Japan. We are grateful to Dr Ogawa for referring the patient and to Drs Nakasato and Hatanaka for helping us to perform magnetoencephalography.

1 Crick F, Koch C. Consciousness and neuroscience. Cerebral Cortex 1998;8:97-107.

2 Schacter DL. Implicit knowledge: new perspectives on unconscious processes. Proc Natl Acad Sci USA 1992;89:

11113-7.

3 Weiskrantz L, Barbur JL, Sahraie A. Parameters affecting conscious versus unconscious visual discrimination with damage to the visual cortex (V1). Proc Natl Acad Sci USA 1995;92:6122-6.

4 Sanders MD, Warrington EK, Marshall J, et al. "Blindsight": Vision in a field defect. Lancet 1974;i:707-8.

5 Shefrin SL, Goodin DS, Aminoff MJ. Visual evoked potentials in the investigation of "blindsight". Neurology 1988;38: 104-9.

6 Weiskrantz L. Blindsight. Oxford: Clarendon Press, 1986.

7 Weiskrantz L, Warrington E, Sanders M, et al. Visual capacity in the hemianopic field following a restricted occipital ablation. Brain 1974;97:709-28.
8 Weiskrantz L. Varieties of residual experience. $O \mathcal{F}$ Exp Psychol 1980;32:365-86.

9 Barbur J, Ruddock K, Waterfield V. Human visual responses in the absence of the geniculo-calcarine projection. Brain 1980;103:905-28.

10 Blythe IM, Kennard C, Ruddock KH. Residual vision in patients with retrogeniculate lesions of the visual pathways. Brain 1987;110:887-905.

11 Ceccaldi M, Mestre D, Brouchon M, et al. Autonomie déambulatoire et perception visuelle du mouvement dans un cas de cécité corticale quasi totale. Rev Neurol (Paris) 1992;148:343-9.

12 Fendrich R, Wessinger CM, Gazzaniga MS. Residual vision in a scotoma: implications for blindsight. Science 1992;258: 1489-91.

13 Adams MJ. Models of word recognition. Cogn Psychol 1979; 11:133-76.

14 Meeres SL, Graves RE. Localization of unseen visual stimuli by humans with normal vision. Neuropsychologia 1990;28: $1231-7$

15 Kolb FC, Braun J. Blindsight in normal observers. Nature 1995;377:336-8.

16 Perenin M. Discrimination of motion direction in perimetrically blind fields. Neuroreport 1991;2:397-400.

17 Stoerig P, Cowey A. Blindsight in man and monkey. Brain Stoerig P, Cowey A.

18 Zeki S, Ffytche DH. The Riddoch syndrome: insights into the neurobiology of conscious vision. Brain 1998;121:2545

19 Marcel AJ. Blindsight and shape perception: deficit of visual consciousness or of visual function? Brain 1998;121:156588.

20 Ffytche D, Howard R, Brammer M, et al. The anatomy of conscious vision: an fMRI study of visual hallucinations. Nature Neuroscience 1998;1:738-42.

21 Nobre A, Allison T, McCarthy G. Word recognition in the human inferior temporal lobe. Nature 1994;372:260-3.

22 Büchel C, Price C, Friston K. A multimodal language region in the ventral visual pathway. Nature 1998;394:274-7. 